\title{
Biopsy of Urethra
}

National Cancer Institute

\section{Source}

National Cancer Institute. Biopsy of Urethra. NCI Thesaurus. Code C51640.

Removal of tissue from the urethra for microscopic examination. 